\title{
Drawing Test Form for Depression: The Development of Drawing Tests for Predicting Depression Among Breast Cancer Patients
}

\author{
Jiyeon $\mathrm{Kim}^{1}$ and Seockhoon Chung ${ }^{2 凶}$ \\ ${ }^{1}$ Department of Art Therapy, Hanyang Cyber University, Seoul, Republic of Korea \\ 2Department of Psychiatry, Asan Medical Center, University of Ulsan College of Medicine, Seoul, Republic of Korea
}

Objective The aim of this study is to develop a drawing test form and it's scoring system for measuring depressive symptoms of breast cancer patients.

Methods We enrolled 95 breast cancer patients in this study which was conducted from September 2016 to May 2018. First, we observed items of the Diagnostic Drawing Series (DDS) which were significantly different between patients with and without depression, and also we reviewed previous studies on the drawing style of Korean women with depression. Second, we developed the Drawing Test Form for Depression (DTF-D) by composition of those drawing styles observed significant items in previous literatures and formative elements of DDS which was significantly different between depressed and non-depressed group. The receiver operating characteristic (ROC) curve analysis was used to define the appropriate score of the DTF-D in accordance with the depression measured with Patient Health Questionnaire-9 (PHQ-9) score $>9$.

Results We developed the DTF-D by compositing 8 items including Harmony, Tilt, Symbol, Space utilization, Surrounded, Description, Pen pressure, and Quality of line. The ROC analysis revealed the optimal cut-off score of 7 points of DTF-D in accordance with the clinical depression (PHQ-9 score $>9$ ) with the area under the receiver operating characteristics curve showed good discrimination $(0.82)$, sensitivity (0.85), and specificity (0.64).

Conclusion The DTF-D is expected to serve as an evaluative projective drawing test from for identifying depression in breast cancer patients.

Psychiatry Investig 2021;18(9):879-888

Keywords Breast cancer; Depression; Distress; Projective drawing test; Art therapy.

\section{INTRODUCTION}

The National Comprehensive Cancer Network announced that it is important to manage cancer patients' psychological symptoms, since their psychological distress can affect treatment outcomes and their quality of life. ${ }^{1}$ In the cancer trajectory, patients often suffer from emotional distress related to the uncertainty of the prognosis, physical symptoms such as fatigue or pain, and psychological symptoms such as insomnia, anxiety, or depression regarding cancer recurrence or metastasis. ${ }^{2,3}$ Particularly, symptoms of depression are commonly observed

Received: February 4, 2021 Revised: June 17, 2021

Accepted: July 19, 2021

$\triangle$ Correspondence: Seockhoon Chung, $\mathrm{MD}, \mathrm{PhD}$

Department of Psychiatry, Asan Medical Center, University of Ulsan College of Medicine, 86 Olympic-ro 43 gil, Songpa-gu, Seoul 05505, Republic of Korea Tel: +82-2-3010-3411, Fax: +82-2-485-8381, E-mail: schung@amc.seoul.kr

(a) This is an Open Access article distributed under the terms of the Creative Commons Attribution Non-Commercial License (https://creativecommons.org/licenses/by$\mathrm{nc} / 4.0$ ) which permits unrestricted non-commercial use, distribution, and reproduction in any medium, provided the original work is properly cited. among cancer patients, and can aggravate their physical symptoms and/or impair physical and social functioning. ${ }^{3}$ It has also been reported that depressed patients may show a lower level of compliance to medical treatment and lower survival rates compared to non-depressed cancer patients. ${ }^{4}$ Therefore, early psychiatric diagnosis and intervention should be conducted for these patients in order to alleviate their psychological distress.

Patients with breast cancer are quite vulnerable to depression. They worry about the prognosis, recurrence, or metastasis, and struggle with the hardships of cancer treatment. Women suffering from breast cancer are required to play their roles as a housewife and mother and fulfill their duties even as they struggle with the difficulties of their illness. Furthermore, for female breast cancer patients, surgical removal of breasts can be psychologically traumatic and emotionally painful, since breasts are not only an important part of the body but are also a symbol of femininity and motherhood. For these reasons, it is even more important to assess their psychiatric symptoms and provide mental health care for them. 
In general, the most appropriate course is that the patients visit a psychiatric clinic where their psychiatric symptoms are evaluated by a trained psychiatrist. However, sometimes it is not easy for these patients to visit a psychiatric clinic due to lack of time, accessibility, or social prejudice. In this situation, selfreport rating scales or questionnaires can be used. Further, psychological projective diagnostic tools can also be used to screen their psychiatric symptoms. In clinical practice, projective tests, like the Rorschach test, can be used by well-trained psychologists to detect patients' psychological problems. One of these projective tests, the House-Tree-Person (HTP) test, can be used by art therapists too. However, while the HTP test is easy to administer, it lacks a standardized scoring system. Therefore, it is meaningful to develop a projective test with a standardized scoring system to detect depression in breast cancer patients. In this context, art therapists attempted to develop a new diagnostic drawing test to overcome the limitations of projective tests. ${ }^{5}$ Among the various drawing tests that were developed, the Diagnostic Drawing Series (DDS) ${ }^{6}$ and the Person Picking an Apple from a Tree (PPAT) ${ }^{7}$ were developed based on the Diagnostic and Statistical Manual of Mental Disorders (DSM). Both these tests have a standardized scoring system for analyzing the drawings. However, they are limited in use as diagnostic tools, since they do not provide an exact cut-off or anchoring point for specific psychiatric symptoms of the patient.

Since art therapists are not trained in psychopathology, such assessment should be performed in collaboration with psychiatrists. In this context, it is meaningful to investigate and determine the anchoring points of the drawing tests in collaboration with psychiatrists. This will help art therapists to appropriately administer the drawing tests to the patients, and guide them to visit psychiatric clinics for professional management of their symptoms by well-trained specialists. Therefore, in this study, we aimed to develop a scoring system for a drawing test to assess depressive symptoms in cancer patients, who need psychological support for their distress during the cancer trajectories, and to explore the anchoring point in the test, which predicts clinical depression.

\section{METHODS}

This prospective study was conducted at the Asan Medical Center from September 2016 to May 2018. We recruited breast cancer patients who volunteered to participate in the study, based on the following inclusion criteria: 1) women with breast cancer aged 20 to 70 years; 2) patients with no major physical illness or major psychiatric problem that may reduce the ability to judge or communicate. We included patients with breast cancer without limitations on the stage of cancer, surgery, or treatment modalities. The study protocol was approved by the
Institutional Review Board (2016-0751) of the Asan Medical Center; all participants signed informed consent forms for participation.

\section{Evaluation}

When the participants visited the Cancer Edu-Info Center of the Asan Medical Center, a psychiatrist (SC) explained the objective of the study to them. They were given the informed consent form, rating scale, and questionnaire inquiring their medical details (age, cancer stage, and current treatment). The patients drew three types of images (free, tree, and feeling) using 12 pastel colors with strong gradients on a quarto paper; this is helpful for maximizing emotional expression, thus helping emotionally suppressed breast cancer patients to express and sublimate their emotions. During the drawing test, the researcher provided relevant topics for each drawing, and refrained from conversation while the participants drew.

\section{Assessment tools}

\section{The Diagnostic Drawing Series \& Drawing Analysis Form}

The DDS was developed ${ }^{8}$ in an attempt to discover a correlation between psychopathology and the formative elements of images, based on diagnostic methods outlined in the DSMIII and DSM-III-R. The DDS utilized 23 formative elements (Color, Idiosyncratic color, Blending, Elements, Line length, Integration, Tree, Depiction, Edges, Image, Enclosure, Pressure, Movement, Space usage, Tilt, Unusual placement, Groundline, People, Animals, Objects, Symbols, Writing, and Landscape) to develop a standardized scoring index, thereby reinforcing subjective errors commonly seen in projective tests. ${ }^{9}$ The DDS has significance as a drawing test tool in that it is the first scientific drawing scale with verified validity and reliability as a supportive evaluation tool for the diagnosis of psychiatric patients. ${ }^{10}$ The advantage of this test is that individuals can comfortably participate in it as the use of pastels promotes relaxation. ${ }^{11}$ After completion of the test, the images drawn by the participants were evaluated based on the Drawing Analysis Form (DAF) developed by Cohen. ${ }^{6,9,12}$

\section{Patient Health Questionnaire-9}

For assessing convergent validity, we used the Patient Health Questionnaire (PHQ)-9. The PHQ-9 was developed to measure patients' depressive symptoms. It has nine items, scored on a scale from 0 to 3 , with a total score ranging from 0 to $27 .{ }^{13}$ A higher score indicates more severe depressive symptoms ( 0 to $4=$ minimal depression, 5 to $9=$ mild depression, 10 to $14=$ moderate depression, 15 to $19=$ moderately severe depression, and $\geq 20=$ severe depression). In this study, we defined a PHQ-9 score of $\geq 10$ as indicating clinical depression. ${ }^{13}$ 


\section{Development of the Drawing Test Form for Depression}

Step 1: Selecting formative elements which are related to depression

In order to develop a drawing test form for evaluating the depressive symptoms of the breast cancer patients, we first observed the differences in the drawing styles of three images (free, tree, and feeling) based on the PHQ-9 scores (score $\leq 9$ vs. $>9$ ).

Step 2: Formative elements reported as associated with depression in the literatures

We reviewed previous studies (published since 2010) ${ }^{14-20}$ on the drawing styles of Korean women with depression, and discovered that the formative elements in the drawings reflected culture and environment, as well as gender-specific characteristics.

Step 3: Evaluating the utility of the scoring system of the DTF-D

Finally, we composited the items observed in step 1 \& 2, and developed the final Drawing Tests Form for Depression. And the validity of the final diagnostic drawing test was evaluated and suggested a cut-off score for clinical depression (PHQ-9 >9).

\section{Ratings of the Drawing Test Form for Depression}

The DFA, developed by Cohen, is able to perform objective evaluations based on 23 items. However, there were limitations in using the DFA as an evaluation tool in art therapy, because the DFA did not provide a quantified scoring method. In this study, we proposed a new evaluation standard for 8 items of DTF-D (Supplementary Material in the online-only Data Supplement), which were observed from step 1 and 2 .

\section{Harmony}

It was reported that patients with depression tend to draw pictures with a lack of integration. ${ }^{21}$ Integration in DDS signifies the overall harmony of the drawing. In this study, we replaced integration of DDS with Harmony. The evaluation criteria of harmony is based on the DFA criteria. If it has good integration and comes well together, it is checked well harmonize. On the other hand, if the drawing lacks integration or if each component is drawn independently so that it lacks visual connection, it is going to be a drawing lacking harmony. Moreover, overly simplified drawings such as stick figures or trees in the shape of a lollipop will also be considered as lacking in harmony.

\section{Tilt}

Drawings that had a tilted vertical-axis to any direction, right and left, were also associated with depression. ${ }^{22}$ Tilt was eval- uated based on the DFA's criteria. However, because the DFA did not provide a clear justification behind the 15-degree evaluation standard, in this study we checked drawings as tilted if the vertical axis was tilted at least 5-degrees to the left or right.

\section{Symbol}

'Symbol' in this study includes the evaluation criteria of landscapes, known to be associated with depression in DFA. In addition to this, it includes objects like the sun, starts, water, animals, people, heart and others that are understood as symbols in art therapy. However, symbolic with personal significance were excluded from the evaluation criteria according to the criteria of DFA that personal symbols are recommended to be excluded from evaluation. Symbol should be possible to identify what it represents, thus excluding unique personal emblems and designs.

\section{Space utilization}

Space usage is also used to evaluate a patient's depression, and it has been reported that patients with depression used very little space. ${ }^{7,21}$ People who are psychologically and emotionally healthy are known to use approximately $2 / 3$ of the surface in general. ${ }^{7}$ Therefore, we decided drawings that used less than $60 \%$ of surface space to be associated with depression. However, since it is difficult to accurately measure $60 \%$ of used surface space, drawings were divided into four blocks across vertical and horizontal axes (16 blocks in total)_drawings that used less than 9 blocks were coded as positive for depression. In this case, empty space comprises approximately $56.2 \%$ of the total surface space. Empty space is defined as space where there is no drawing or only colored in without any expression of a specific object, in accordance with DFA standard.

\section{Surrounded}

Similar the previous study, ${ }^{22}$ enclosure in DFA was associated with depression. The DFA has separate check boxes to evaluate enclosure and surrounded, but we unite those two items as a single item of Surrounded, since symbolic meanings of both items were similar in art therapy psychological and physical defense. ${ }^{23}$ The label becomes surrounded to imply both meaning, enclosure and surrounded.

\section{Description}

Description is an indicator to evaluate the detailedness of the drawing. Drawings that lack detailed portrayals indicate lack of energy and signs of depression. ${ }^{24} \mathrm{Park}^{17}$ and $\mathrm{Choi}^{18}$ found that the drawings of people with depression lacked detail or form.

\section{Pen pressure}

Called pressure in DFA, refers to the pressure of the hand 
when drawing and can be categorized as low, medium, and strong; the evaluation criteria for pen pressure follows that of DDS.

\section{Quality of line}

The quality of line is an important criterion to assess patient's status. Patients with depression usually use short lines ${ }^{14}$ or faint lines with low pen pressure. ${ }^{23}$ Therefore, this study used short lines or disconnected lines as a criteria to evaluate depression.

\section{Example of ratings of the Drawing Tests for Depression}

Figure 1 shows an example of ratings of the Drawing Test for Depression. The subject agreed that we use her drawings in the manuscript. Case 1: a 49-year-old woman who was assessed as having depression; Patients Health Questionnaire-9 items scale score was 14 and the Drawing Test Form for Depression score was 11. First, a free drawing (Figure 1A); there is a considerable distance between the two trees (Harmony, 1 point), a trunk of the tree was drawn with disconnected short lines (Quality of line, 1 point), pen pressure of the drawing is weak (Pen pressure, 1 point). The drawing is divided by the sea and land with a fence which can be understood as a symbol of depression and lack of sociality (Symbol, 1 point), and the fence creates a divided boundary of land (Surrounded, 1 point). Second, a tree drawing (Figure 1B); similar to the free drawing, pen pressure is weak (Pen pressure, 1 point), the tree was drawn with disconnected short lines (Quality of line, 1 point), and the falling nuts on the ground symbolizes feelings of hopelessness (Symbol, 1 point). Third, a feeling drawing (Figure 1C), weak pen pressure (Pen pressure, 1 point), heart- shaped materials as a symbol of love (Symbol, 1 point), and the letters inside of the heart was rated as enclosed (Surrounded, 1 point).

\section{Statistical analysis}

Statistical analyses were performed using SPSS version 21.0 for Windows (IBM Corp., Armonk, NY, USA). The clinical characteristics were summarized as mean \pm standard deviation.
The level of significance for all analyses was defined as $\mathrm{p}<0.05$ (two-tailed), and the chi-square test was used for betweengroup analysis of the categorical variables. Receiver operating characteristic (ROC) analysis was carried out to explore the appropriate cut-off point for the drawing test in accordance with the PHQ-9 scores.

\section{RESULTS}

Ninety-five breast cancer patients participated in this study; the majority of them $(n=36,37.9 \%)$ were in their 40 s. Among participants, 6 (6.3\%) were stage 0, 32 (33.7\%) were stage I, 33 (34.8\%) were stage II, 18 (18.9\%) were stage III, and 6 (6.5\%) were stage IV. Most patients received surgical treatment $(\mathrm{n}=90$, 94.7\%), with some undergoing chemotherapy and hormonal therapy during the course of this study. The demographics of the participants are outlined in Table 1.

\section{Step 1: selecting formative elements which are related to depression}

Table 2 shows the differences in formative elements of the DDS in three drawings between depressed and non-depressed patients. We just present the elements which were significantly different between two groups (Integration, Tilt, Landscape, Space usage, and Enclosure). The degree of Integration refers to the overall harmony of the drawing, and the proportion of disintegration in free drawing was higher among depressed patients compared to non-depressed $\left(\chi^{2}=7.075, \mathrm{p}=0.03\right)$. Tilt refers to a condition when the vertical axis of the drawing is tilted more than 15 degrees to the surface, and this element showed a significant difference just in free drawing $\left(\chi^{2}=7.564, \mathrm{p}=0.01\right)$; tilt was more prevalent in the depressed than in the non-depressed group (17.5\% vs. $43.8 \%$ ). Landscape refers to the drawing include water, sun, star, animal and other objects. It showed a significant difference in only free drawing $\left(\chi^{2}=11.323, \mathrm{p}=0.01\right)$. Space usage was divided into four stages based on the rate of surface use. This element showed a significant difference just

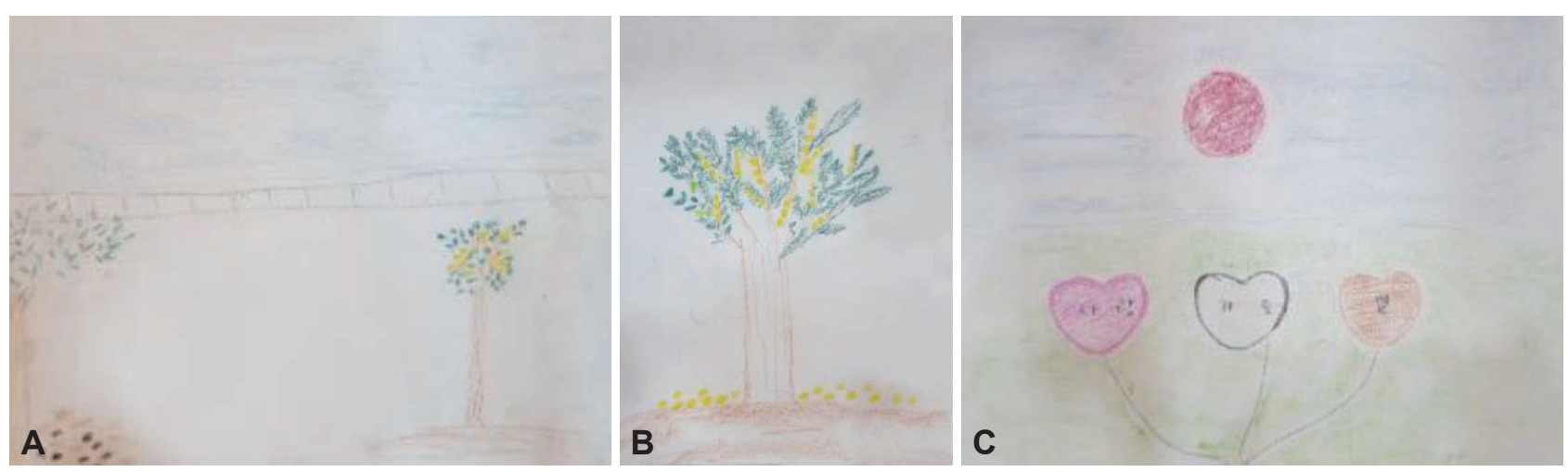

Figure 1. Analysis of the drawings of a subject using the Drawing Test Form for Depression. A: A free drawing. B: A tree drawing. C: A feeling drawing. 
Table 1. Demographic characteristics of the participants

\begin{tabular}{lc}
\hline \multicolumn{1}{c}{ Variables } & Total participants (N=95) \\
\hline $\begin{array}{l}\text { Age (years) } \\
\leq 39\end{array}$ & $21(22.1)$ \\
$40-49$ & $36(37.9)$ \\
$50-59$ & $28(29.5)$ \\
$\geq 60$ & $10(10.5)$ \\
Cancer stage & \\
0 & $6(6.3)$ \\
I & $32(33.7)$ \\
II & $33(34.8)$ \\
III & $18(18.9)$ \\
IV & $6(6.3)$ \\
Treatment history & \\
Radiation therapy & $51(53.7)$ \\
Hormones therapy & $26(27.4)$ \\
Chemotherapy & $21(22.1)$ \\
History of surgical operation & $90(94.7)$ \\
Others & $8(8.4)$ \\
\hline
\end{tabular}

in free drawing $\left(\chi^{2}=8.337, \mathrm{p}=0.04\right)$. Enclosure refers to the closure of the drawing. The presence of closure showed statistical significance when analyzed against the score of depression, in all of three drawings. In free drawing, $15.6 \%$ of the participants with depression drew an enclosure, while $44.4 \%$ of the participants without depression drew it $\left(\chi^{2}=7.775, p=0.006\right)$. In tree drawing, $6.2 \%$ of patients with depression and $12.7 \%$ of patients without depression drew it $\left(\chi^{2}=0.937, \mathrm{p}=0.006\right)$. However, feeling drawing showed contrasting results, where $30.2 \%$ of the non-depressed patients drew an enclosure, while $53.1 \%$ of the depressed patients drew $\left(\chi^{2}=4.576, \mathrm{p}=0.04\right)$.

\section{Step 2: formative elements reported as associated with depression in the literatures}

We reviewed previously published studies about the relationship between psychiatric symptoms and drawing response characteristics of breast cancer patients, which were available from the Korea Education and Research Information Service; for this study, we only considered papers published after 2010. We excluded studies that were conducted on teenagers or male patients. We identified 10 studies that targeted adult female patients; of these studies, four were published in 2010, two in 2011, two in 2012, one in 2014, and one in 2016. We confirmed that depression was related to Integration, ${ }^{14}$ Tilt, ${ }^{15}$ Symbols, ${ }^{15,16,18,19}$ Space usage, ${ }^{17,19}$ Depiction, ${ }^{16,18}$ Pressure,,${ }^{15,16}$ Color, ${ }^{17,20}$ and Line length. ${ }^{15,16}$ Combining the literature and the results of the DDS test in this study, we confirmed that 9 elements, including Integration, Tilt, Symbols, Enclosure, Space usage, Depiction, Pres- sure, Color, Line length, were related to depression (Table 3). Based on the results of this study, a scoring system was developed for the drawing test for depression (Supplementary Material in the online-only Data Supplement). Finally, we decided to remove the element of Color, which displayed an excessively biased ratio in drawing responses of the breast cancer patients, from the final DTF-D.

Evaluation of the drawings included the degree of expression in showing harmony and description, with quality of lines, enclosure, tilt, and symbolism evaluated based on whether or not they were present in the drawing. The standard for use of space was also established, where, if the participant used more than $70 \%$ of the provided area, she was evaluated as emotionally stable. However, since $70 \%$ of the paper was difficult to define quantitatively, the actual evaluation involved dividing the paper into 16 equal pieces. A lack of space usage was concluded if the drawing was present in fewer than 10 of these pieces (i.e., $62.5 \%$ of the total surface). If the lines were short, lacking pen pressure, or if no lines were used in the drawing, it was deemed to be a drawing response characteristic of a depressed patient, and was scored as 1 . Based on the study by Kim et al., ${ }^{22}$ breast cancer patients with depression showed mostly moderate pen pressure, unlike psychiatric patients with depression. Pen pressure was classified into three levels with the following scores: weak pen pressure $=1$, medium pen pressure $=0.5$, and strong pen pressure $=0$.

\section{Step 3: evaluating the utility of the scoring system of the DTF-D}

From step $1 \& 2$, we selected 9 items of Integration, Tilt, Symbol, Space usage, Depiction, Pressure, Color, and Quality of lines (Table 3). In this study, we finalized the Drawing Test Form for Depression editing the concepts as "Harmony, Tilt, Symbol, Space utilization, Surrounded, Description, Pen pressure, and Quality of line." The color was not included in the final form, since more than $85 \%$ of patients used more than four colors.

The total score of the DTF-D was significantly correlated with the PHQ-9 score $(r=0.45, p<0.01)$, and a significant correlation was observed in each free, tree, and feeling drawings (Table 4). From the ROC analysis (Figure 2), the optimal cut-off for the drawing test for depression for PHQ-9 $>9$ was 7, with an area under the curve of 0.82 , a sensitivity of 0.85 , and specificity of 0.64 .

\section{DISCUSSION}

In this study, we attempted to develop and validate a standardized scoring system for the drawing test for depression. The drawing test form for depression consists of 8 items including Harmony, Tilt, Symbol, Space utilization, Surrounded, De- 
Table 2. The graphic elements of the DDS and depression scores

\begin{tabular}{|c|c|c|c|c|}
\hline Variables & $\begin{array}{l}\text { Not depressed }(\mathrm{N}=63) \\
(\text { PHQ-9 } \leq 9)\end{array}$ & $\begin{array}{c}\text { Depressed }(\mathrm{N}=32) \\
(\mathrm{PHQ}-9>9)\end{array}$ & Total $(\mathrm{N}=95)$ & $\chi^{2}$ test, $p$-value \\
\hline \multicolumn{5}{|l|}{ DDS: integration } \\
\hline Free drawing & & & & $\chi^{2}=7.075, \mathrm{p}=0.03^{*}$ \\
\hline Disintegration & $1(1.6)$ & $5(15.6)$ & $6(6.3)$ & \\
\hline Integration & $51(81.0)$ & $22(68.8)$ & $73(76.9)$ & \\
\hline Impoverish & $11(17.4)$ & $5(15.6)$ & $16(16.8)$ & \\
\hline Tree drawing & & & & $\chi^{2}=1.868, p=0.40$ \\
\hline Disintegration & $4(6.3)$ & $2(6.2)$ & $6(6.3)$ & \\
\hline Integration & $56(88.9)$ & $26(81.3)$ & $82(86.3)$ & \\
\hline Impoverish & $3(4.8)$ & $4(12.5)$ & $7(7.4)$ & \\
\hline Feeling drawing & & & & $\chi^{2}=0.294, p=0.86$ \\
\hline Disintegration & $15(23.8)$ & $7(21.9)$ & $22(23.2)$ & \\
\hline Integration & $38(60.3)$ & $21(65.6)$ & $59(62.1)$ & \\
\hline Impoverish & $10(15.9)$ & $4(12.5)$ & $14(14.7)$ & \\
\hline \multicolumn{5}{|l|}{ DDS: tilt } \\
\hline Free drawing & & & & $\chi^{2}=7.564, p=0.01^{*}$ \\
\hline No & $52(82.5)$ & $18(56.3)$ & $70(73.7)$ & \\
\hline Yes & $11(17.5)$ & $14(43.7)$ & $25(26.3)$ & \\
\hline Tree drawing & & & & $\chi^{2}=0.285, p=0.68$ \\
\hline No & $59(93.7)$ & $29(90.6)$ & $88(92.6)$ & \\
\hline Yes & $4(6.3)$ & $3(9.4)$ & $7(7.4)$ & \\
\hline Feeling drawing & & & & $\chi^{2}=0.229, p=0.75$ \\
\hline No & $55(87.3)$ & $29(90.6)$ & $84(88.4)$ & \\
\hline Yes & $8(12.7)$ & $3(9.4)$ & $11(11.6)$ & \\
\hline \multicolumn{5}{|c|}{ DDS: landscape (symbol) } \\
\hline Free drawing & & & & $\chi^{2}=11.323, p=0.01^{*}$ \\
\hline Landscape & 39 (61.9) & $14(43.8)$ & $53(55.8)$ & \\
\hline With water & $11(17.5)$ & $5(15.6)$ & $16(16.8)$ & \\
\hline Water only & $5(7.9)$ & $0(0.0)$ & $5(5.3)$ & \\
\hline None & $8(12.7)$ & $13(40.6)$ & $21(22.1)$ & \\
\hline Tree drawing & & & & $\chi^{2}=0.224, p=0.89$ \\
\hline Landscape & $39(61.9)$ & $19(59.4)$ & $58(61.1)$ & \\
\hline With water & $5(7.9)$ & $2(6.3)$ & $7(7.4)$ & \\
\hline Water only & $0(0.0)$ & $0(0.0)$ & $0(0.0)$ & \\
\hline None & $19(30.2)$ & $11(34.4)$ & $30(31.6)$ & \\
\hline Feeling drawing & & & & $\chi^{2}=6.390, p=0.94$ \\
\hline Landscape & $6(9.5)$ & $2(6.3)$ & $8(8.4)$ & \\
\hline With water & $4(6.3)$ & $5(15.6)$ & $9(9.5)$ & \\
\hline Water only & $8(12.7)$ & $0(0.0)$ & $8(8.4)$ & \\
\hline None & $45(71.4)$ & $25(78.1)$ & $70(73.7)$ & \\
\hline
\end{tabular}


Table 2. The graphic elements of the DDS and depression scores (continued)

\begin{tabular}{|c|c|c|c|c|}
\hline Variables & $\begin{array}{c}\text { Not depressed }(\mathrm{N}=63) \\
(\text { PHQ-9 } \leq 9)\end{array}$ & $\begin{array}{c}\text { Depressed }(\mathrm{N}=32) \\
\quad(\text { PHQ-9>9) }\end{array}$ & Total $(\mathrm{N}=95)$ & $\chi^{2}$ test, $\mathrm{p}$-value \\
\hline \multicolumn{5}{|l|}{ DDS: space usage } \\
\hline Free drawing & & & & $\chi^{2}=8.337, p=0.04^{*}$ \\
\hline $1 \%-33 \%$ & $0(0.0)$ & $4(12.5)$ & $4(4.2)$ & \\
\hline $34 \%-66 \%$ & $3(4.8)$ & $1(3.1)$ & $4(4.2)$ & \\
\hline $67 \%-99 \%$ & $48(76.2)$ & $21(65.6)$ & $69(72.7)$ & \\
\hline Full & $12(19.0)$ & $6(18.8)$ & $18(18.9)$ & \\
\hline Tree drawing & & & & $\chi^{2}=0.135, p=0.99$ \\
\hline $1 \%-33 \%$ & $14(22.2)$ & $8(25.0)$ & $22(23.2)$ & \\
\hline $34 \%-66 \%$ & $6(9.5)$ & $3(9.4)$ & $9(9.5)$ & \\
\hline $67 \%-99 \%$ & $36(57.2)$ & $18(56.2)$ & $54(56.8)$ & \\
\hline Full & $7(11.1)$ & $3(9.4)$ & $10(10.5)$ & \\
\hline Feeling drawing & & & & $\chi^{2}=4.328, p=0.23$ \\
\hline $1 \%-33 \%$ & $2(3.2)$ & $3(9.4)$ & $5(5.3)$ & \\
\hline $34 \%-66 \%$ & $9(14.3)$ & $2(6.3)$ & $11(11.6)$ & \\
\hline $67 \%-99 \%$ & $46(73.0)$ & $21(65.5)$ & $67(70.5)$ & \\
\hline Full & $6(9.5)$ & $6(18.8)$ & $12(12.6)$ & \\
\hline \multicolumn{5}{|l|}{ DDS: enclosure } \\
\hline Free drawing & & & & $\chi^{2}=7.775, p=0.006$ \\
\hline No & $35(55.6)$ & $27(84.4)$ & $62(65.3)$ & \\
\hline Yes & $28(44.4)$ & $5(15.6)$ & $33(34.7)$ & \\
\hline Tree drawing & & & & $\chi^{2}=0.937, \mathrm{p}=0.006$ \\
\hline No & $55(87.3)$ & $30(93.8)$ & $85(89.5)$ & \\
\hline Yes & $8(12.7)$ & $2(6.2)$ & $10(10.5)$ & \\
\hline Feeling drawing & & & & $\chi^{2}=4.576, p=0.04^{*}$ \\
\hline No & $44(69.8)$ & $15(46.9)$ & $59(62.1)$ & \\
\hline Yes & $19(30.2)$ & $17(53.1)$ & $36(37.9)$ & \\
\hline
\end{tabular}

Values are presented as number (\%). ${ }^{*} \mathrm{p}<0.05 ;{ }^{\dagger} \mathrm{p}<0.01$. DDS, Diagnostic Drawing Series; PHQ-9, Patient Health Questionnaire-9

scription, Pen pressure, and Quality of line. We found a cutoff point of 7 on this drawing test form to indicate depression, which was validated against a score for clinical depression defined as PHQ-9 $>9$.

Based on the 23 formative elements of the DDS, depression in the breast cancer patients showed a correlation with the number of colors used, degree of integration, closure status, degree of space usage, tilt, and background. However, as more than $85 \%$ of the participants used more than four colors, this element showed too much bias to be meaningful. Whereas, integration showed a higher prevalence in the non-depressed group than in the depressed group. The concept that a psychologically healthier mind demonstrates more harmony and creativity than the mind of a depressed person was directly reflected in the drawings.

Enclosure indicates a mental isolation from the outside world. ${ }^{25}$ In this study, participants with depression showed less closure in free drawing but more in feeling drawing. Closure may not have been apparent in free drawing due to a characteristic (expression suppression) of breast cancer patients. They may repress their thought and emotion in the first drawing because of high tension to new environment. Yet, because feeling drawing is the last drawing of DDS and use an abstract subject, participants may reduce the tension and express their thought and emotion freely.

The non-depressed patients exhibited a higher space usage rate than the depressed group in free drawing. A larger number of people in the depressed group used less than $33 \%$ of the 
space provided, which was consistent with two previous studies. ${ }^{21,24}$ From the art therapy perspective that the paper space used by participants represents their world, ${ }^{23}$ it may be concluded that depressed people who have lost interest in life showed reduced willingness to fully utilize the space provided for their drawings.

Tilt only differed between the two groups in free drawing $\left(\chi^{2}=7.564, p=0.01\right)$. The use of tilt was more prevalent in the

Table 3. Graphic elements significantly related to depression

\begin{tabular}{ll}
\hline \multicolumn{1}{c}{ Diagnostic Drawing Series } & In previous literature \\
\hline Depression & \\
Integration & Integration $^{14}$ \\
Tilt & Tilt $^{15}$ \\
Landscape & Symbol $^{15,16,18,19}$ \\
Space usage & Space usage $^{17,19}$ \\
Enclosure & - \\
- & Depiction \\
- & Pressure $^{15,16}$ \\
- & Color $^{17,20}$ \\
- & Quality of lines \\
\hline
\end{tabular}

Table 4. Correlation coefficients between the PHQ-9 scale score and the modified drawing test score

\begin{tabular}{lc}
\hline & PHQ-9 (N=95) \\
\hline Total score on the modified drawing test & $0.45^{\dagger}$ \\
Free drawing score & $0.31^{\dagger}$ \\
Tree drawing score & $0.26^{*}$ \\
Feeling drawing score & $0.22^{\dagger}$ \\
${ }^{*} \mathrm{p}<0.05 ;{ }^{\dagger} \mathrm{p}<0.01$. PHQ-9, Patient Health Questionnaire-9
\end{tabular}

depressed than in the non-depressed group. This was in line with the results of Mankckam and Sajani, ${ }^{26}$ which found more tilt in the drawing response characteristics of depressed teenagers, and complemented Park's opinion ${ }^{27}$ that people who are intimidated and shy exhibit tilt. Tilt can be understood as a depiction of participants' intimidated psychological state as a result of their cancer diagnosis.

Depressed patients' expression in relation to background was also visible only in free drawing, where the non-depressed group used all categories of background, including background, water drawing (such as lakes or rivers), and water-only drawing (where water is the main focus) more often than the depressed group. The depressed group more often produced a blank drawing. Our finding that the non-depressed group used more water or water-only drawings contradicted the results of Cohen et al., ${ }^{12}$ who found a higher prevalence of water-only drawings among depressed patients. Even though water has been understood as indicating sadness in art therapy, water also symbolizes fecundity in myth, which may explain our finding. The greater prevalence of the blank drawing category among breast cancer patients with depression can be understood as a reflection of the psychological desires of breast cancer patients, who expressed a desire for social isolation and emotional suppression by not including a subject in their drawings.

Based on the literature and the results of the DDS, a lack of harmony and description, quality of lines, presence of enclosure and division, usage of symbolism, use of less space, and weak pen pressure were selected as indicators to derive a scoring index for identifying depression in breast cancer patients. The total evaluation score of the three drawings was 24 , with a cut-off value for depression as 7 (AUC 0.82 , sensitivity 0.85 ,
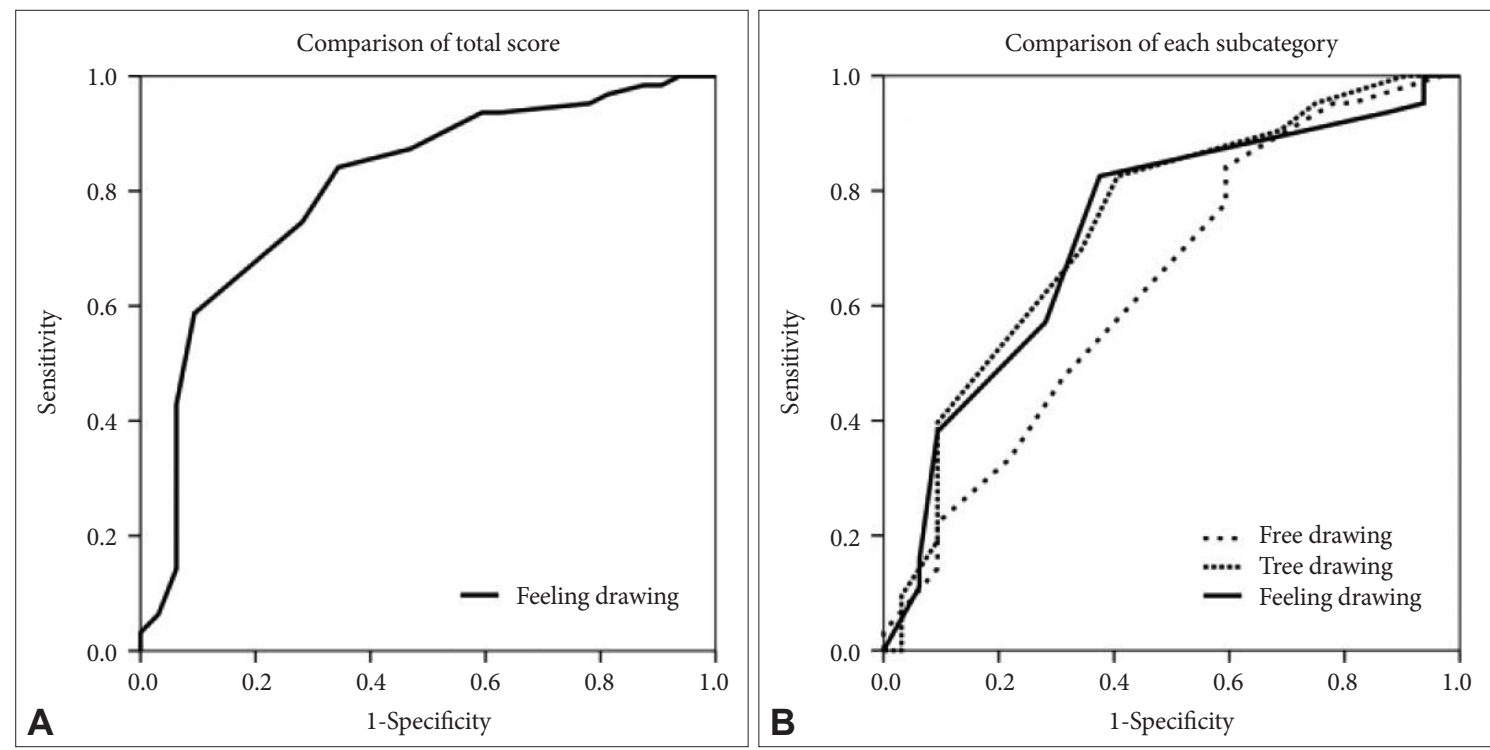

Figure 2. The receiver operating characteristic (ROC) curves for depression. A: ROC curve for total score of the modified drawing test for depression. B: ROC curves for each subcategory of the modified drawing test for depression. 
and specificity 0.64 ). Thus, this study standardized an evaluation method for breast cancer patients' drawing by suggesting clear scoring criteria and developing a scoring index. Therefore, it is meaningful in providing a threshold for evaluating depression in breast cancer patients using a drawing test. Our results indicate that drawing tests could potentially be used as supportive diagnostic tools for evaluating depression in breast cancer patients in the future.

Nevertheless, the number of participants was limited, and the evaluation of depression depended on a self-report survey rather than a diagnosis by a professional psychiatrist. Additionally, although this study suggested a universal, quantitative evaluation method for drawings by breast cancer patients, the evaluation of the drawings was still subjective. Therefore, there is a need to continue studying drawing response characteristics in breast cancer patients to obtain a more specific standard for evaluating such drawings in future. A more detailed standard for evaluation and a quantitative evaluation method will help drawing tests overcome a lack of consistency and gain more value as a supportive tool in evaluating psychiatric symptoms in breast cancer patients in the medical field.

We attempted to develop a drawing test form for diagnosing depression among breast cancer patients. Although it still is not easy to apply this test in clinical practice, the findings provide a new direction for future research and practice, which can be helpful for assessing depressive symptoms in breast cancer patients.

\section{Supplementary Materials}

The online-only Data Supplement is available with this article at https://doi.org/10.30773/pi.2021.0044.

\section{Availability of Data and Material}

The datasets generated or analyzed during the study are available from the corresponding author on reasonable request.

\section{Conflicts of Interest}

The authors have no potential conflicts of interest to disclose.

\section{Author Contributions}

Conceptualization: Jiyeon Kim, Seockhoon Chung. Data curation: Jiyeon Kim, Seockhoon Chung. Formal analysis: Jiyeon Kim, Seockhoon Chung. Investigation: Jiyeon Kim, Seockhoon Chung. Methodology: Jiyeon Kim, Seockhoon Chung. Software: Jiyeon Kim, Seockhoon Chung. Validation: Jiyeon Kim, Seockhoon Chung. Writing_-original draft: Jiyeon Kim, Seockhoon Chung. Writing-review \& editing: Jiyeon Kim, Seockhoon Chung.

\section{ORCID iDs}

Jiyeon Kim https://orcid.org/0000-0001-7406-3176

Seockhoon Chung https://orcid.org/0000-0002-9798-3642

\section{Funding Statement}

None.

\section{REFERENCES}

1. Riba MB, Donovan KA, Andersen B, Braun I, Breitbart WS, Brewer BW, et al. Distress management, version 3.2019, NCCN clinical practice guidelines in oncology. J Natl Compr Canc Netw 2019;17:12291249.

2. Sharpe L, Curran L, Butow P, Thewes B. Fear of cancer recurrence and death anxiety. Psychooncology 2018;27:2559-2565.

3. Yeo S, Lee J, Kim K, Kim HJ, Chung S. Depression, rather than cancerrelated fatigue or insomnia, decreased the quality of life of cancer patients. Cancer Res Treat Cancer Res Treat 2021;53:641-649.

4. Haskins CB, McDowell BD, Carnahan RM, Fiedorowicz JG, Wallace $\mathrm{RB}$, Smith BJ, et al. Impact of preexisting mental illness on breast cancer endocrine therapy adherence. Breast Cancer Res Treat 2019;174: 197-208.

5. Fowler J, Ardon A. Diagnostic drawing series and dissociative disorders: a Dutch study. Art Psychother 2002;29:221-230.

6. Cohen B, Mills A, Kijak A. An introduction to the diagnostic drawing series: a standardized tool for diagnostic and clinical use. J Am Art Therapy Assoc 1994;11:105-110.

7. Gantt L, Anderson F. The formal elements art therapy scale: a measurement system for glodal variables in art. Art Therapy 2009;26:124129.

8. Mills A, Cohen B, Meneses J. Reliability and validity tests of the diagnostic drawing series. Art Psychother 1993;20:83-88.

9. Kessler K. A study of the diagnostic drawing series with eating disordered patients. Art Therapy 1994;1:116-118.

10. Brooks S. Tools of the Trade: A Therapist's Guide to Art Therapy Assessments. Springfield: Charles C Thomas publisher; 2004.

11. Jeon S. The Healing Factors and Media of Art Therapy. Seoul: Hanampc; 2011.

12. Cohen B, Hammer J, Singer S. The Diagnostic Drawing Series: A systematic approach to art therapy evaluation and research. Art Psychother 1988;15:11-21.

13. Kroenke K, Spitzer RL, Williams JB. The PHQ-9: validity of a brief depression severity measure. J Gen Intern Med 2001;16:606-613.

14. Lee SH, Eo EK, Kim KS. A study on how effectively the characteristic of the drawing in DDS can detect women's depression tendency. J Art Psychother 2014;10:145-167.

15. Joo JH. Study of the Response to Tree-Drawing Tests According to the Symptoms of Depression in Middle Aged Woman [Master]. Pyeongtaek: Pyeongtaek University; 2011.

16. Park SY. The Study on Relationship between Cultural Adaptation Stress and Depression with Response Characteristic of 'Person in the Rain' Drawing Test of Marital Emigration Women [Master]. Daegu: Daegu University; 2010.

17. Park HS. The Characteristics of Responses to PPAT(Person Picking an Apple from a Tree) Assessment [Master]. Seoul: Seoul Womens University; 2012.

18. Choi JS. A study on Postpartum Depression and Reaction Cheracteriscs on the 'Persons in the Rain' Drawing [Master]. Daegu: Daegu University; 2010.

19. Kim SM. Response Characteristic of 'Person in the Rain' Drawing Test on Post-Traumatic Stress and Depression of North Korean Refugees [Master]. Daegu: Daegu University; 2011.

20. Choi HW. Research on Depression and FSA Response Characteristics of Office Workers [Master]. Daegu: Youngnam University; 2016.

21. Kang H. The Characteristics of Responses to PPAT (The Person Picking an Apple from a Tree) Assessment according to the Level of Depression and Self-esteem in Pregnant Women. Seoul: Ehwa Womens University; 2018.

22. Kim J, Youn S, Choi B, Jung KH, Ahn SD, Hwang SY, et al. Psychological distress and drawing tests among women with breast cancer. J Korean Med Sci 2018;33:e140.

23. Shin M, Kim S, Kim Y, Kim J, Kim H, Kim J. Diagnosis and Under- 
standing of Children's Art Through Picture: Focusing on HTP and KFD. Seoul: Hackgisa; 2002.

24. Choi J, Choi S. A study on PPAT response by the levels of depression and self-efficacy of early adult. Kor J Art Therapy 2010;17:1165-1190.

25. Reynolds C. A quick scoring guide to the interpretation of children's kinetic family drawings (KFD). Psychol Sch 1978;15:489-492.
26. Mankckam L, Sajani V. Elements of drawing and depression among adolescents in Kerala, South India: an explorative study using a person picking a mango from a tree. SIS J Proj Psy Ment Health 2016;23:41-46.

27. Park H. Understanding of Diagnosis in Art Therapy. Paju: Yangseowon; 2009. 


\section{Supplementary Material}

\section{Drawing Test Form for Depression (DTF-D)}

This test contains 8 questions that will be used to evaluate depression. Observe closely and answer the questions that follow. While answering the questions, judge the drawing based on its overall mood. After evaluation of the three drawings (free, tree, and emotion) is completed, please rate them according to the evaluation method (EM) of each drawing element. The total score is 24. A score of $\geq 7$ is considered as depression.

\section{Total score}

\section{Harmony}

This question is for proper consonance between elements and/or images in the drawing. The relevance or graphic consistency between the subject and drawing is important. Improper distance between the items in the drawing indicates a lack of harmony. "Harmony" refers to "integration" in the Drawing Test Form for Depression DAF.

\begin{tabular}{|l|c|c|}
\hline \multicolumn{1}{|c|}{ Questions } & Yes & No \\
\hline Lack of graphical or thematic harmony. & & \\
\hline There is isolation or considerable distance between items. & & \\
\hline Drawing with defensive characteristics (e.g., keyhole tree, lollipop tree, stick man) & & \\
\hline (EM) If at least one of the answers is marked as "Yes", allot a score of "1." & 1 & 0 \\
\hline
\end{tabular}

\section{Tilt}

A slope of more than 5 degrees to the left or right. The use of tools to measure the slope is recommended because it is difficult to gauge the slope with the naked eye.

\begin{tabular}{|l|c|c|}
\hline \multicolumn{1}{|c|}{ Questions } & Yes & No \\
\hline The vertical axis is tilted more than 5 degrees to the left or right. & & \\
\hline (EM) If at least one of the answers is marked as "Yes", allot a score of "1" & 1 & 0 \\
\hline
\end{tabular}

\section{Symbol}

Conventional representation of a single image such as a mark or sign (it should not be a personal idea). It includes all the symbolic items incorporating the criteria of "landscape" in the DAF.

\begin{tabular}{|l|c|c|}
\hline \multicolumn{1}{|c|}{ Questions } & Yes & No \\
\hline The image used is represented conventionally or larger than its original meaning. & & \\
\hline A mark or sign that is generally called a symbol in art therapy. & & \\
\hline (EM) If at least one of the answers is marked as "Yes", allot a score of "1"” & 1 & 0 \\
\hline
\end{tabular}

\section{Space utilization}

To evaluate this, the drawing sheet is visually divided into four equal parts horizontally and vertically. A total of 16 blocks are made. At least 9 blocks should be filled with drawings. It does not count if a block has been filled only with color without any drawing. "Space utilization" is the concise version of "Space usage" in the DAF.

\begin{tabular}{|l|c|c|}
\hline \multicolumn{1}{|c|}{ Questions } & Yes & No \\
\hline $\begin{array}{l}\text { Even if more than nine blocks are painted, most of them are meaningless drawings that } \\
\text { have been painted with the side of the pastel. }\end{array}$ & & \\
\hline Less than nine blocks are filled with a drawing. & 1 & 0 \\
\hline (EM) If at least one of the answers is marked as "Yes," allot a score of "1." & \\
\hline
\end{tabular}




\section{Surrounded}

When a single-colored form or line completely surrounds an item. However, it does not have to be perfectly enclosed. "Surrounded" includes the criteria of "enclosure" in the DAF.

\begin{tabular}{|l|c|c|}
\hline \multicolumn{1}{|c|}{ Questions } & Yes & No \\
\hline The form or line encloses an item that is alive. & & \\
\hline The form or line surrounds an item that is alive. & & 1 \\
\hline (EM) If at least one of the answers is marked as "Yes," allot a score of "1." & & \\
\hline
\end{tabular}

\section{Description}

If the drawing is expressed in detail or not. Look carefully for a lack of description, distortion of size, omission, and emphasis in the drawing. If less than three colors are used, mark it as "lack of description." "Description" including the criteria of "depiction" in the DAF has a larger concept of expression.

\begin{tabular}{|l|c|c|}
\hline \multicolumn{1}{|c|}{ Questions } & Yes & No \\
\hline A lack of description. & & \\
\hline Any distortion of size in the drawing (small size). & & \\
\hline Any omitted parts (except the nose and ears of people). & & 1 \\
\hline It is hard to recognize the form because of inaccuracy. & & 0 \\
\hline (EM) If at least one of the answers is marked as "Yes", allot a score of “1." & \\
\hline
\end{tabular}

\section{Pen pressure}

It refers to the amount of pressure that was applied when drawing. It has three levels: dim, medium, and strong. Dim is when the white color of the paper is visible. Medium is when the color has been painted well overall. Strong is when it is hard to discern the white part of the paper and when the color was painted very thick with the pastel. "Pen pressure" includes the criteria of "pressure" in the DAF.

\begin{tabular}{|c|c|c|c|}
\hline Questions & Dim & Medium & Strong \\
\hline Read the above-mentioned instructions and choose any one: Dim, Medil & & & \\
\hline (EM) If at least one of the answers is marked as "Yes," allot a score of " 1. " & 1 & 0 & 0 \\
\hline
\end{tabular}

\section{Quality of line}

It is a graphic element that expresses an individual's personality and feelings. The questions should be answered after checking the condition of the line. "Quality of line" includes the criteria of "Line length" in the DAF.

\begin{tabular}{|l|c|c|}
\hline \multicolumn{1}{|c|}{ Questions } & Yes & No \\
\hline The line drawn is pale. & & \\
\hline Drawing without lines using only facets of the pastel. & & \\
\hline A long line that is formed using unconnected short lines. & & 1 \\
\hline (EM) If at least one of the answers is marked as "Yes", allot a score of "1." & & 0 \\
\hline
\end{tabular}

